\title{
A SIMPLE CONDITION ENSURING THE ARENS REGULARITY OF BILINEAR MAPPINGS
}

\author{
NILGÜN ARIKAN
}

\begin{abstract}
We give a simple criterion for certain Banach algebras to be Arens regular, which applies in particular to the algebras $l^{1}$ with pointwise multiplication, $L^{\infty}(G)$, where $G$ is a compact group with convolution, and the trace-class algebra. This criterion is best established in the more general context of the regularity of bilinear maps, and depends on the existence of extensions of such maps.
\end{abstract}

1. Let $X, Y, Z$ be normed spaces and $m: X \times Y \rightarrow Z$ be a bounded bilinear mapping. Arens gives the two natural extensions $m^{* * *}$ and $m^{t^{* *} t}$ of $m$ onto $X^{* *} \times Y^{* *} \rightarrow Z^{* *}$ in [1]. These are constructed by forming in turn the following bilinear mappings:

$$
\begin{aligned}
& Z^{*} \times X \rightarrow Y^{*}:(f, x) \rightarrow{ }_{x} f, \text { where }{ }_{x} f(y)=f(m(x, y)), \\
& Y^{* *} \times Z^{*} \rightarrow X^{*}:(G, f) \rightarrow f_{G} \text {, where } f_{G}(x)=G\left({ }_{x} f\right),
\end{aligned}
$$

and

$$
X^{* *} \times Y^{* *} \rightarrow Z^{* *}:(F, G) \rightarrow F \circ G, \text { where } F \circ G(f)=F\left(f_{G}\right) .
$$

We call $F \circ G=m^{* * *}(F, G)$ the first extension of $m$. Similarly, we form the next three mappings:

$$
\begin{aligned}
Z^{*} \times Y & \rightarrow X^{*}:(f, y) \rightarrow f_{y}, \quad \text { where } f_{y}(x)=f(m(x, y)), \\
X^{* *} \times Z^{*} & \rightarrow Y^{*}:(F, f) \rightarrow{ }_{F} f, \quad \text { where }{ }_{F} f(y)=F\left(f_{y}\right),
\end{aligned}
$$

and

$$
X^{* *} \times Y^{* *} \rightarrow Z^{* *}:(F, G) \rightarrow F * G, \text { where } F * G(f)=G\left({ }_{F} f\right)
$$

and call $F * G=m^{t * * t}(F, G)$ the second extension of $m$. We call $m$ regular if $\circ$ and * coincide on $X^{* *} \times Y^{* *}$. This is equivalent to saying that the double limits $\lim _{i} \lim _{j} f\left(m\left(x_{i}, y_{j}\right)\right), \lim _{j} \lim _{i} f\left(m\left(x_{i}, y_{j}\right)\right)$ are equal whenever they both exist for bounded sequences $\left(x_{i}\right) \in X,\left(y_{j}\right) \in Y$ and $f \in Z^{*}$; see [3]. The latter is known as the Double Limit Criterion. Then the regularity of a normed algebra $A$ is defined to be the regularity of its algebra multiplication when considered as a bilinear mapping. Let $F$ and $G$ be elements of $A^{* *}$, the second dual of $A$. By Goldstine's Theorem, pp. 424-425 of [4], there exist nets $\left(x_{i}\right)$ and $\left(y_{j}\right)$ in $A$ so that $F=w^{*} \lim _{i} e\left(x_{i}\right)$ and $G=w^{*} \lim _{j} e\left(y_{j}\right)$ where $e: A \rightarrow A^{* *}$ denotes the canonical embedding of $A$ into $A^{* *}$

Received by the editors December 8, 1980.

1980 Mathematics Subject Classification. Primary 46J10, $22 \mathrm{C} 05$.

Key words and phrases. Arens regularity, bilinear mappings, second duals of Banach algebras.

(C)1982 American Mathematical Society 0002-9939/81/0000-0793/\$02.50 
as a normed space. So it is easy to see that $\lim _{i} \lim _{j} f\left(m\left(x_{i}, y_{j}\right)\right)=F \circ G(f)$ and $\lim _{j} \lim _{i} f\left(m\left(x_{i}, y_{j}\right)\right)=F * G(f)$ for $f \in A^{*}$.

In this paper we shall prove that a bilinear map is regular if it factors through some other bilinear mapping. We shall also prove that irregular algebras may have dense subalgebras which are regular when given a different norm.

Definition 1. Let $X, Y, Z, W$ all be normed spaces and $h$ be a continuous linear mapping of $Y$ into $W$ so that the following diagram

$\begin{array}{cc}X \times Y & \\ 1_{X} \times h \downarrow & \searrow m_{1} \\ X \times W & \nearrow m_{2} \\ & \end{array}$

commutes, i.e., $m_{1}(x, y)=m_{2}(x, h(y))$ for all $x \in X, y \in Y$. Then we say that $m_{1}$ factors through $m_{2}$.

TheOREM 2. Let $m_{1}: X \times Y \rightarrow Z$ be a bounded bilinear mapping. If there exists a bounded bilinear mapping $m_{2}: X \times W \rightarrow Z$ such that $m_{1}$ factors through $m_{2}$ and such that the continuous linear mapping $h: Y \rightarrow W$ of the above diagram has the property that $h\left(Y_{1}\right)$ is $\sigma\left(h(Y), W^{*}\right)$-compact where $Y_{1}$ is the unit ball of $Y$, then $m_{1}$ is regular.

Proof. We will prove that for sequences $\left(x_{i}\right)$ and $\left(y_{j}\right)$ in the unit ball of $X$ and $Y$ respectively and $f \in Z^{*}$,

$$
\lim _{i} \lim _{j} f\left(m_{1}\left(x_{i}, y_{j}\right)\right)=\lim _{j} \lim _{i} f\left(m_{1}\left(x_{i}, y_{j}\right)\right)
$$

when both double limits exist. It is straightforward to see that $f\left(m_{1}\left(x_{i}, y_{j}\right)\right)=$ $e\left(x_{i}\right) * e\left(h\left(y_{j}\right)\right)(f)$ by using $f\left(m_{1}\left(x_{i}, y_{j}\right)\right)=f\left(m_{2}\left(x_{i}, y_{j}\right)\right)$. Now by the BanachAlaoglu Theorem $\left(e\left(x_{i}\right)\right)$ has a subnet weak* converging to some $F$ in $X^{* *}$ and because $h\left(Y_{1}\right)$ is weakly compact in $W,\left(h\left(y_{j}\right)\right)$ has a subnet which converges weakly to $h(y)$ for some $y \in Y_{1}$. Since the limits of the subnets are the same as the original limits, we may replace the original nets by the subnets and assume that $F$ is the weak* limit of $\left(e\left(x_{i}\right)\right)$ and $h(y)$ is the weak limit of $\left(h\left(y_{j}\right)\right)$. Now consider the following diagram (in which arrows represent limits, not mappings):

$$
\begin{array}{ccc}
e\left(x_{i}\right) * e\left(h\left(y_{j}\right)\right)(f) & \underset{i \downarrow}{\mathrm{III}} & e\left(x_{i}\right) * e(h(y))(f) \\
i \downarrow \mathrm{I} & & i \downarrow \mathrm{II} \\
F * e\left(h\left(y_{j}\right)\right)(f) & \underset{\mathrm{IV}}{\overrightarrow{\mathrm{IV}}} & F * e(h(y))(f)
\end{array}
$$

It is easy to observe that $*$ is weak* continuous on the right. So the horizontal limits III and IV follow as $e(h(y))$ is the weak* limit of $\left(e\left(h\left(y_{j}\right)\right)\right)$. For the vertical limits we recall that, for a Banach algebra $B, x \mapsto x * e(y)$ is weak* continuous on $B^{* *}$ for each $y$ in $B$; see [3]. Now the result follows. 
Theorem 3. Let $A, B, X, Z$ all be normed spaces. Suppose that $A^{*}=B$ and there is a continuous linear injection $h: B \rightarrow A$ such that

$$
\phi(h(\psi))=\psi(h(\phi))
$$

for all $\phi, \psi \in A^{*}$ is satisfied. If there exist bounded bilinear mappings $m_{1}: X \times B \rightarrow Z$ and $m_{2}: X \times A \rightarrow Z$ such that $m_{1}$ factors through $m_{2}$ then $m_{1}$ is regular.

Proof. We know $B_{1}$ is $\sigma(B, A)$ compact because $B=A^{*}$. From condition (1) we get $h:\left(B, w^{*}\right) \rightarrow(A, w)$ is continuous. So it is clear that $h\left(B_{1}\right)$ is $\sigma\left(h(B), A^{*}\right)$ compact. Therefore the result follows from Theorem 2 .

Now, we are going to give several concrete examples to Theorem 3 as corollaries.

Corollary 4. Let $X, Z$ be normed spaces. Suppose there exist bounded bilinear mappings

$$
m_{1}: X \times l^{1} \rightarrow Z \text { and } m_{2}: X \times c_{0} \rightarrow Z
$$

such that $m_{1}$ factors through $m_{2}$ when $h$ is taken to be the natural inclusion of $l^{1}$ in $c_{0}$. Then $m_{1}$ is regular. Furthermore, the natural extension of $m_{1}$ onto $X^{* *} \times\left(l^{1}\right)^{* *} \rightarrow Z^{* *}$ is regular.

Proof. Since we have $\phi(h(\psi))=\Sigma_{n} \phi_{n} \psi_{n}=\psi(h(\phi))$ for $\phi=\left(\phi_{n}\right), \psi=\left(\psi_{n}\right) \in$ $\left(c_{0}\right)^{*} \cong l^{1}$, the condition (1) of Theorem 3 is satisfied. So the regularity of $m_{1}$ follows.

We now prove that $m_{1}^{* * *}$ is regular. It is easy to see that $m_{1}^{* * *}$ factors through $m_{2}^{* * *}$ since $F \circ G(f)=F \circ h^{* *}(G)(f)$ for $F \in X^{* *}, G \in\left(l^{1}\right)^{* *}, f \in Z^{*}$ and $h^{* *}$ : $\left(l^{1}\right)^{* *} \rightarrow\left(c_{0}\right)^{* *}$ is the second adjoint mapping of $h: l^{1} \rightarrow c_{0}$. We now recall from [2] or [3] that

$$
\left(l^{1}\right)^{* *}=e\left(l^{1}\right) \oplus e\left(c_{0}\right)^{\perp} .
$$

Then a simple observation shows that $h^{* *}$ is the mapping from $\left(l^{1}\right)^{* *}$ onto $l^{1}$ given by $h^{* *}(F)=F_{1}$, for $F=\left(F_{1}, F_{2}\right) \in\left(l^{1}\right)^{* *}$. Furthermore, we observe that

$$
\begin{aligned}
\sigma\left(h^{* *}\left(\left(l^{1}\right)^{* *}\right),\left(c_{0}\right)^{* * *}\right) & =\sigma\left(l^{1},\left(l^{1}\right)^{* *}\right)=\sigma\left(c_{0}, e\left(l^{1}\right) \oplus e\left(c_{0}\right)^{\perp}\right) \uparrow^{1} \\
& =\left(c_{0}, l^{1}\right) \uparrow^{1}=\sigma\left(l^{1}, l^{1}\right) .
\end{aligned}
$$

Now the regularity of $m_{1}^{* * *}$ follows from Theorem 2 since on the unit ball of $l^{1}$, $\sigma\left(l^{1}, l^{1}\right)$ coincides with $\sigma\left(l^{1}, c_{0}\right)$.

COROLlary 5. Let $X=[0,1]$ be the unit interval and $\eta$ be the Lebesgue measure on the real line. Then $L^{\infty}=L^{\infty}(X)$ is the Banach space of all essentially bounded Lebesgue measurable complex functions on $X$ with the usual norm and $I_{82}^{1}=L^{1}(X)$ is the Banach space of all complex valued Lebesgue measurable functions on $X$ with its usual norm. Then $L^{1}$ is a commutative Banach algebra with the convolution multiplication defined by

$$
(f * g)(x)=\int_{0}^{x} f(x-t) g(t) d t
$$

for $x \in X, f, g \in L^{1}$. There is a natural inclusion $h$ from $L^{\infty}$ into $L^{1}$ for which $L^{\infty}$ is a subalgebra of $L^{1}$ with a different norm and also $L^{\infty}(X) \cong L^{1}(X)^{*}$. Now convolution 
defines a bounded bilinear mapping $m_{1}: L^{1} \times L^{\infty} \rightarrow L^{1}$ so that $m_{1}$ factors through the algebra multiplication on $L^{1}$. Since the condition (1) of Theorem 3 is also satisfied for $A=L^{1}$ and $B=L^{\infty}$ the regularity of $m_{1}$ follows from the same theorem.

Corollary 6. Let $C(X)$ be the Banach algebra of all continuous bounded functions on $X=[0,1]$ with the supremum norm and the convolution multiplication defined by (2). Then convolution defines a bounded bilinear mapping

$$
C(X) \times L^{\infty}(X) \rightarrow C(X):(f, g) \rightarrow f * g
$$

which is regular.

Proof. Recall from $\$ 31$ of [5] that $x \rightarrow f * g(x)$ is continuous for $f \in L^{\infty}$ and $g \in L^{1}$ (and so, a fortiori, for $f \in L^{\infty}$ and $g \in L^{\infty}$ or $g \in C(X)$ ). Hence the existence of the bounded bilinear mapping $C(X) \times L^{1}(X) \rightarrow C(X)$ with convolution multiplication makes the proof clear.

COROLlary 7. The Banach algebra $C(X)$ is regular with convolution multiplication.

Proof. Since $C(X)$ is a closed subalgebra of $L^{\infty}(X)$ the result is clear from the corollary on p. 312 of [3].

For $X=[0,1]$, we have proved by Corollary 7 that $C(X)$ is regular with convolution multiplication in the form of (2). We are now going to prove that $C(X)$ has regular second and fourth duals.

Let $\lambda$ be an element of $C(X)^{*} \cong M(X)$. Then by the Lebesgue Decomposition Theorem there is a unique representation of $\lambda$ in the form $\lambda=\sigma+\nu$ where $\sigma$ is absolutely continuous with respect to $\eta$ (denoted by $\sigma \ll \eta$ ) and $\nu$ is singular with respect to $\eta$ (denoted by $\nu \perp \eta$ ). As it is easy to see that $\|\lambda\|=\|\sigma\|+\|\nu\|$ we have

$$
M(X)=L^{1}(X) \oplus M_{s}(X), \quad \text { where } M_{s}(X)=\{\mu \in M(X): \mu \perp \eta\} .
$$

Now by a theorem due to Kakutani $M(X)^{*}\left(\cong C(X)^{* *}\right)$ is an $M$-space and of the form $M(X)^{*}=L^{\infty}(X) \times M_{s}(X)^{*}$. Obviously, $L^{\infty}(X)$ is a subspace of $M(X)^{*}$ and the multiplication in $M_{s}(X)^{*}$ will be found by considering its quotient algebras $L^{\infty}(\lambda)$ for each $\lambda \in M_{s}(X)$.

First let $f$ and $g$ be in the unit ball of $L^{\infty}(X)$. By Goldstine's Theorem there are sequences $\left(f_{n}\right)$ and $\left(g_{m}\right)$ in the unit ball of $C(X)$ with $\left\|f_{n}\right\| \leqslant\|f\|,\left\|g_{m}\right\| \leqslant\|g\|$ and $e\left(f_{n}\right) \stackrel{w^{*}}{\rightarrow} f, e\left(g_{m}\right) \stackrel{w^{*}}{\rightarrow} g$. Then

$$
\left(f_{n} * g_{m}\right)=\int_{0}^{1} \int_{0}^{1}\left(\chi f_{n}\right)(x-t)\left(\chi g_{m}\right)(t) d t \cdot d x \text { by (2) }
$$

where $\chi$ is the characteristic function of $X$. By taking iterated limits and using the Dominated Convergence Theorem and Fubini's Theorem we get that

$$
\lim _{n} \lim _{m} \eta\left(f_{n} * g_{m}\right)=\int_{0}^{1} \int_{0}^{1}(\chi f)(x-t)(\chi g)(t) d t \cdot d x=(f * g)(\eta)
$$

and also $\lim _{m} \lim _{n} \eta\left(f_{n} * g_{m}\right)=(f * g)(\eta)$.

Now let $f$ and $g$ be in $M_{s}(X)^{*}$ and $\nu \in M_{s}(X)$. We want to find $(f * g)(\nu)$. Since $L^{\infty}(\nu)$ is a quotient of $M_{s}(X)^{*}$ there is a function $(f * g)^{\sim}$ in $L^{\infty}(\nu)$ such that 
$\nu\left((f * g)^{\sim}\right)=(f * g)(\nu)$. Similarly, there are functions $\tilde{f}, \tilde{g}$ in $L^{\infty}(\nu)$ with $\nu(\tilde{f})=$ $f(\nu), \nu(\tilde{g})=g(\nu)$. Assume $\nu$ is positive. Then $X$ is the union of disjoint sets $A$ and $B$ for which $\nu(A)=0, \eta(B)=0$. By assuming $\tilde{f}=0$ on $A$ we get $\eta(\{x: \tilde{f}(x) \neq 0\})=0$ and hence $\tilde{f}$ is $\eta$-measurable. Thus $\tilde{f}=0$ a.e. $(\eta)$. Similarly, $\tilde{g}=0$ a.e. $(\eta)$. Put $\gamma=\nu+\eta$. We have $\tilde{f}=0$ on $A$ and $|\tilde{f}| \leqslant\|\tilde{f}\|_{\infty}$ on $B$ (this can be done by altering $\tilde{f}$ on a set of $\nu$-measure 0$)$. From Lusin's Theorem there exists a sequence $\left(f_{n}\right)$ in $C(X)$ with

$$
\tilde{f}(x)=\lim _{n} f_{n}(x) \text { a.e. }(\gamma) \text {. }
$$

Consequently, $f_{n} \rightarrow \tilde{f}$ a.e. $(\nu)$ and $f_{n} \rightarrow \tilde{f}$ a.e. $(\eta)$. Hence $\lim _{n} f_{n}=0$ a.e. $(\eta)$ since $\tilde{f}=0$ a.e. $(\eta)$. Similarly, there exists a sequence $\left(g_{m}\right)$ in $C(X)$ with $\lim _{m} g_{m}=0$ a.e. $(\eta)$. From the regularity of $C(X)$ and using the Dominated Convergence Theorem we get

$$
\begin{aligned}
(f * g)(\nu)=\nu\left((f * g)^{\sim}\right) & =\lim _{n} \lim _{m} \nu\left(f_{n} * g_{m}\right) \\
= & \lim _{m} \lim _{n} \nu\left(g_{m} * f_{n}\right)=\lim _{m} \int_{0}^{1} \lim _{n}\left(\chi f_{n}\right)(t)\left[\int_{0}^{1}\left(\chi g_{m}\right)(x-t) d \nu(x)\right] \cdot d t=0 .
\end{aligned}
$$

So we have $(f * g)(\nu)=0$ for all $f, g \in M_{s}(X)^{*}$. Thus for $u=\left(\Pi_{1} u, \Pi_{2} u\right)$ and $v=\left(\Pi_{1} v, \Pi_{2} v\right)$ in $M(X)^{*}$ with $\Pi_{1} u, \Pi_{1} v \in L^{\infty}(X)$ and $\Pi_{2} u, \Pi_{2} v \in M_{s}(X)^{*}$ and $\lambda=(\sigma, v) \in M(X)$ with $\sigma \in L^{1}(X), \nu \in M_{s}(X)$, the Arens product of $u$ and $v$ is

$$
\begin{aligned}
(u \circ v)(\lambda) & =(u * v)(\lambda)=\sigma\left(\Pi_{1} u * \Pi_{1} v\right)+\nu\left(\Pi_{2} u * \Pi_{2} v\right) \\
& =\sigma\left(\Pi_{1}(u * v)\right)+0=\left(\Pi_{1}(u * v), 0\right)(\sigma+v) \\
& =\left(\Pi_{1}(u * v), 0\right)(\lambda) .
\end{aligned}
$$

COROLlaRY 8. $L^{\infty}(X)$ is regular with convolution multiplication.

Proof. Since the algebra multiplication of $L^{\infty}(X)$ given by convolution factors through the bilinear mapping $L^{\infty} \times L^{1} \rightarrow L^{\infty}, L^{\infty} \cong\left(L^{1}\right)^{*}$ and the condition (1) of Theorem 3 is satisfied for $A=L^{1}, B=L^{\infty}$, the result follows from it.

COROllary 9. The second dual of $C(X), C(X)^{* *} \cong M(X)^{*}$, is regular.

Proof. The result follows since $M(X)^{*}$ is regular if and only if $L^{\infty}(X)$ is regular.

Since the multiplication on $M(X)^{*} \cong L^{\infty}(X) \times M_{s}(X)^{*}$ is zero in the second coordinate the multiplication on $M(X)^{* * *} \cong L^{\infty}(X)^{* *} \times M_{s}(X)^{* * *}$ has the same property. Therefore " $M(X)^{* * *} \cong C(X)^{* * * *}$ is regular if and only if $L^{\infty}(X)^{* *}$ is regular."

COROLlaRY 10. $L^{\infty}(X)^{* *}$ is regular.

Proof. We have already observed in the course of the proof of Corollary 8 for the bounded bilinear mappings

$$
m_{1}: L^{\infty} \times L^{\infty} \rightarrow L^{\infty} \text { and } m_{2}: L^{\infty} \times L^{1} \rightarrow L^{\infty}
$$

given by convolution $m_{1}$ factors through $m_{2}$ by $h: L^{\infty} \rightarrow L^{1}$ the natural inclusion. Then the first extension $m_{1}^{* * *}$ of $m_{1}$ factors through $m_{2}^{* * *}$, the first extension of $m_{2}$, 
by $h^{* *}$, the second adjoint mapping of $h$. So it is enough to prove that $h^{* *}\left(\left(L^{\infty}\right)_{1}^{* *}\right)$ is $\sigma\left(h^{* *}\left(\left(L^{\infty}\right)^{* *}\right),\left(\left(L^{1}\right)^{* *}\right)^{*}\right)$-compact. Now consider the diagram

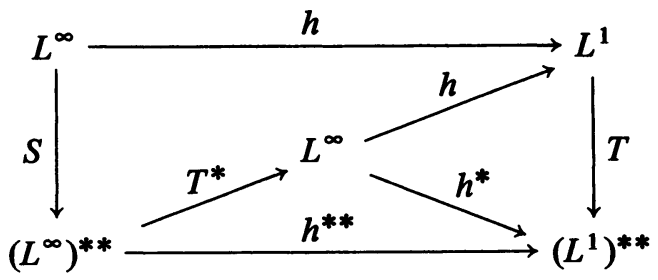

where $S$ and $T$ denote the canonical embeddings of $L^{\infty}$ and $L^{1}$ into their second duals respectively, and $h^{*}$ is the first adjoint mapping of $h$. The diagram is commutative. The subspace $h^{* *}\left(\left(L^{\infty}\right)^{* *}\right)$ of $\left(L^{1}\right)^{* *}$ is $L^{\infty}$ regarded as a subset of $\left(L^{1}\right)^{* *}$. So it is enough to prove that $\left(L^{\infty}\right)_{1}$ is $\sigma\left(L^{\infty},\left(L^{\infty}\right)^{* *}\right)$-compact. Since $L^{\infty}$ is dense in $L^{1}$,

$$
\sigma\left(L^{\infty},\left(L^{\infty}\right)^{* *}\right)=\sigma\left(L^{1},\left(L^{\infty}\right)^{* *}\right) \uparrow_{L^{\infty}}=\sigma\left(L^{1}, L^{\infty}\right) \uparrow_{L^{\infty}}
$$

The latter follows since $L^{1} \subseteq\left(L^{1}\right)^{* *} \cong\left(L^{\infty}\right)^{*}$ and, for $u$ in $\left(L^{\infty}\right)^{* *}, f \in L^{1}, u(T(f))$ $=T^{*}(u)(f)$. By recalling that $\left(L^{\infty}\right)_{1}$ is $\sigma\left(L^{\infty}, L^{\infty}\right)$-compact and applying Theorem 2 the proof is completed.

2. In this section $G$ will denote a compact Hausdorff group and $\mu$ will denote the Haar measure on $G$. We will see that the group algebra $L^{1}(G)$ has a regular dense subalgebra in a different norm. The convolution multiplication on $L^{1}(G)$ is denoted by $*$.

Proposition 11. The bounded bilinear mapping

$$
L^{1}(G) \times L^{\infty}(G) \rightarrow L^{1}(G):(f, g) \rightarrow f * g
$$

is regular.

Proof. It is easy to see that the given bilinear mapping is continuous and it clearly factors through the algebra multiplication of $L^{1}$ given by convolution. Now the result follows from Theorem 3 for $A=L^{1}(G), B=L^{\infty}(G)$ and $X=Z=L^{1}(G)$.

Because $L^{\infty}(G)$ is a dense subset of $L^{1}(G)$ the above result is worth noting since the multiplication in the group algebra $L^{1}(G)$ of a locally compact Hausdorff group $G$ is regular if and only if $G$ is finite; see [7].

PROPOSITION 12. The bounded bilinear mapping

$$
L^{\infty}(G) \times L^{\infty}(G) \rightarrow C(G):(f, g) \rightarrow f * g
$$

is regular.

Proof. We have seen in the proof of Corollary 6 that $f * g \in C(G)$ for $f, g \in$ $L^{\infty}(G)$. We also have that the given bilinear mapping is continuous since

$$
|f * g(x)| \leqslant \int_{G}\left|f\left(x y^{-1}\right)\right| \cdot|g(y)| d \mu(y) \leqslant\|f\|_{\infty}\|g\|_{\infty} .
$$


As $\|f\| \leqslant\|f\|_{\infty}$ for any $f \in L^{\infty}(G)$, the existence of the bounded bilinear mapping $L^{\infty}(G) \times L^{1}(G) \rightarrow C(G):(f, g) \rightarrow f * g$ also follows. Now the rest follows by applying Theorem 3 for $A=L^{1}(G), B=L^{\infty}(G), X=L^{\infty}(G)$ and $Z=C(G)$.

COROLlaRY 13. For a compact group $G, L^{\infty}(G)$ is regular with convolution multiplication.

Proof. This is clear.

3. In this section we will prove that the Banach algebra of trace-class operators on a Hilbert space $H$ is regular. We denote by $L(H), C L(H)$ and $T L(H)$ the set of bounded linear operators, compact operators and trace-class operators on $H$, respectively. We recall that

$$
T L(H)=\left\{S \in L(H):\|S\|_{1}=\operatorname{tr}|S|=\sum_{n=1}^{\infty}\left(\psi_{n},|S| \psi_{n}\right)<\infty\right\},
$$

where $\left(\psi_{n}\right)_{n=1}^{\infty}$ is an orthonormal basis for $H$.

THEOREM 14. The Banach algebra of trace-class operators on a Hilbert space $H$ is Arens regular.

Proof. We recall from [6] that

(i) $T L(H)$ is a two-sided ideal in $L(H)$.

(ii) $\|S\| \leqslant\|S\|_{1}$ for all $S$ in $T L(H)$ ( \|\| is the uniform operator norm on $L(H))$.

(iii) Every $S$ in $T L(H)$ is compact.

(iv) For each $S \in T L(H)$ define $S(K)=\operatorname{tr}(S K)$ for all $K \in C L(H)$. Then the mapping

$$
T L(H) \rightarrow[C L(H)]^{*}: S \mapsto \operatorname{tr}(S .)
$$

is an isometric isomorphism of $T L(H)$ onto $[C L(H)]^{*}$.

(v) For all $S \in T L(H)$ and $T \in L(H), \operatorname{tr}(S T)=\operatorname{tr}(T S)$.

Now the existence of the continuous linear injection $h: T L(H) \rightarrow C L(H)$ follows from (ii) and (iii). The operator multiplication in $L(H)$ defines the two bilinear mappings

$$
m_{1}: T L(H) \times T L(H) \rightarrow T L(H) \text { and } m_{2}: T L(H) \times C L(H) \rightarrow T L(H) .
$$

The latter is clear from (i). Also $m_{1}$ factors through $m_{2}$. Now we put $A=C L(H)$ and $X=Z=B=T L(H)$. So it follows that $A^{*}=B$ and condition (1) of Theorem 3 are satisfied by (iv) and (v) above, respectively. Hence the result follows from Theorem 3.

I would like to thank Professor J. S. Pym for his advice and interest in this work.

\section{REFERENCES}

1. R. Arens, The adjoint of a bilinear operation, Proc. Amer. Math. Soc. 2 (1951), 839-848.

2. P. Civin and B. Yood, The second conjugate of a Banach algebra as an algebra, Pacific J. Math. 11 (1961), 847-870.

3. J. Duncan and S. A. R. Hosseiniun, The second dual of a Banach algebra, Proc. Roy. Soc. Edinburgh Sect. A 84 (1979), 309-325. 
4. N. Dunford and J. T. Schwartz, Linear operators. I, Interscience, New York, 1958.

5. L. H. Loomis, An introduction to abstract harmonic analysis, Van Nostrand, Princeton, N. J., 1953.

6. M. Reed and B. Simon, Methods of modern mathematical physics. I, Academic Press, New York, 1972.

7. N. J. Young, The irregularity of multiplication in group algebras, Quart. J. Math. Oxford Ser. (2) 24 (1973), 59-62.

Department of Pure Mathematics, University of Sheffield, Sheffield S10 2TN, United KINGDOM

Current address: Department of Mathematics, Hacettepe University, Beytepe, Ankara, Turkey 